\title{
On the dependence of third- and fourth-order moments on stability in the turbulent boundary layer
}

\author{
A. Maurizi \\ Institute of Atmospheric Sciences and Climate via Gobetti 101, I-40129 Bologna, Italy \\ Received: 12 October 2005 - Revised: 27 January 2006 - Accepted: 30 January 2006 - Published: 27 April 2006 \\ Part of Special Issue "Turbulent transport in geosciences"
}

\begin{abstract}
Analysis of published data on the third- and fourth-order moments of vertical velocity in the atmospheric boundary layer is performed, and it is shown that turbulence is non-Gaussian even when almost symmetric. It is also proposed that the relationship between third- and fourth-order moments depends on stability. New data from a wind tunnel experiment roughly support this hypothesis. The analysis presented can explain some differences among datasets, and provides a starting point for investigation on the dependence of third- and fourth-order moments on stability.
\end{abstract}

\section{Introduction}

Data on the third- and fourth-order moments of turbulent velocities in boundary layers have been collected for many years, not only in the atmospheric boundary layer (ABL), where their evaluation is somewhat difficult (Lenschow et al., 1994), but also in laboratory experiments, where the relationship between odd-order moments and their upper next evenorder has been studied (see Durst et al., 1987, for instance).

The role of third order moments has long been recognised, at least in the Convective Boundary Layer (CBL), as connected to non-local transport properties (Wyngaard and Weil, 1991). As regards the fourth-order moments, recent studies further point out the inaccuracy of the so-called Millionshchikov, or Quasi-Normal, hypothesis (Losch, 2004), and some interpretations have been formulated based on simplified parameterisations in terms of third-order moments (Tampieri et al., 2000; Gryanik and Hartman, 2002; Alberghi et al., 2002). This approach can be viewed in a more general framework in which odd-order moments are connected algebraically to their next even-order in two-value processes (Kendall and Stuart, 1977; Chatwin and Sullivan,

Correspondence to: A. Maurizi

(a.maurizi@isac.cnr.it)
1990; Lewis et al., 1997). However there is an open question as to whether fourth-order cumulants can be assumed zero for vanishing skewness. The underlying assumption is that turbulence can be assumed as a Gaussian process when symmetric.

In the following, a further interpretation of algebraic models is given in order to highlight some properties of these parameterisations. Furthermore, new data from a wind tunnel experiment on neutral boundary layer will be analysed to verify whether they support the hypothesis of Gaussianity for symmetric turbulence, to what extent and under which conditions.

\section{Algebraic parameterisations of kurtosis}

In the study of high-order moments, particular interest is devoted to the normalised moments, i.e., skewness $(S)$ and kurtosis $(K)$ and relationships between them. Maurizi and Tampieri (1999), and subsequently Tampieri et al. (2000), collected and analysed data from literature on horizontal and vertical velocity skewness and kurtosis, considering a variety of turbulence generation mechanisms. Furthermore, recent papers focus attention in particular on the CBL: new measurements from aircraft (Gryanik and Hartman, 2002, GH hereafter) and with remote sensing acoustic techniques (SODAR) (Alberghi et al., 2002, AMT hereafter) have been presented.

Different simple parameterisations have been proposed for the $S$ - $K$ relationship. Tampieri et al. (2000), first, and subsequently Maurizi and Lorenzani (2001), proposed a parameterisation based on the observation that a statistical limit exists in the $S-K$ space (Kendall and Stuart, 1977), namely

$K \geq K_{\lim }=S^{2}+1$.

This limit shapes the structure of the $S$ - $K$ space. In fact, the existence of a statistical limit for the relationship between

Published by Copernicus GmbH on behalf of the European Geosciences Union and the American Geophysical Union. 


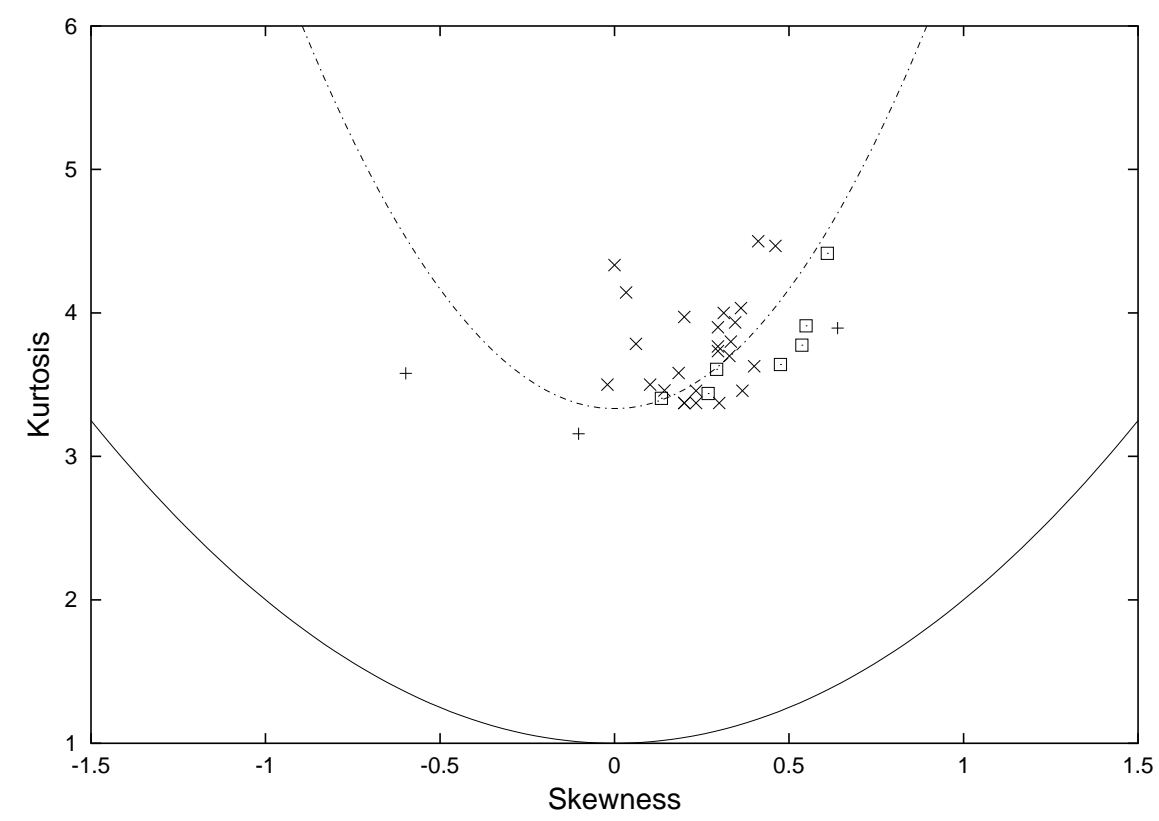

Fig. 1. Skewness and kurtosis for shear dominated boundary layers (Tampieri et al., 2000). Symbols refer to different dataset collected from literature. Continuous line represents the statistical limit, while the dashed line is Eq. (3) with $\alpha_{0}=3.3$ (reprinted courtesy of SGEditoriali, Padova, Italy).

skewness and kurtosis, makes the Millionshchikov hypothesis very limited in the range of skewness values. In the $S$ $K$ space the Millionshchikov hypothesis fails for $|S|>\sqrt{2}$. Moreover, since Eq. (1) is valid for a very peculiar probability density function (linear combination of two delta functions, i.e., a two-value process), a range $S_{\text {lim }}<S<\sqrt{2}$ can be expected to exists in which the probability density function cannot be representative of any turbulence process (Maurizi and Lorenzani, 2000). In other words, if one considers the Millionshchikov hypothesis as a zero-order assumption for fourth-order moments, in a "curved" space, it can be restated in a different way.

If the pair $(S, \tilde{K})$ with $\tilde{K}=K\left(S^{2}+1\right)^{-1}$ is taken as the natural coordinate system for the $S-K$ space, the "equivalent" Millionshchikov hypothesis reads as

$K=3\left(S^{2}+1\right)$.

Assuming a free parameter, the simplest model (zero-order) based on this observation can be built assuming a constant $\tilde{K}$ and, therefore,

$K=\alpha_{0}\left(S^{2}+1\right)$.

Fitting Eq. (3) to data, Tampieri et al. (2000) found, for the vertical velocity component, $\alpha_{0}=3.3$ for shear dominated boundary layers (see Fig. 1) and $\alpha_{0}=2.5$ in the CBL. Although Fig. 1 shows a large scatter, it is worth noting that the lowest value of $K$ is markedly larger than 3. AMT, adding new data to the Tampieri et al. (2000) dataset, confirmed the CBL result, giving $\alpha_{0}=2.4$. The quantity and quality of data collected force the conclusions that, at least in the convective boundary layer, turbulence is far from being Gaussian.

$\mathrm{GH}$ found that a mass-flux assumption for the CBL exactly results in $K=K_{\text {lim }}$ (see Eq. 1) as a relationship between $S$ and $K$. In fact, it is known that this relationship only holds for two-value processes. They used, as a generalisation, the form

$K=\alpha_{0}\left(\beta S^{2}+1\right)$.

It is worth noting that Lewis et al. (1997) proposed a relationship of the same form for concentration data, based on the ideas expressed by Chatwin and Sullivan (1990). It can be observed that this expression cannot be strictly represented in terms of any order expansion in the $(S, \tilde{K})$ space. However, it can be considered as a modified zero-order model. The need to make this modified version stems from the fact that the GH dataset shows strictly leptokurtic cases (see GH, their Fig. 10), forcing them to assume Gaussianity for symmetric distributions. The reliability of this assumption seems to be confirmed by the fact that the GH model compares well to the data, in terms of the explained variance (see $\mathrm{GH}$, their Figs. 8 and 9).

However, this assumption is in clear contrast with respect to the AMT dataset ${ }^{1}$ which contains a great deal of data (more than 50\%) with $K<3$.

One of the possible sources of contrast is that the data in GH refer to a specific turbulent boundary-layer structure

\footnotetext{
${ }^{1}$ The two articles appeared at almost the same time, therefore GH were not aware of the AMT data.
} 
(cold air outbreak over the ocean), and does not represent the large variety of data covered by the AMT dataset, which collects measurements from presumably different environmental conditions. Moreover, if included in Fig. 3 of AMT, GH data would be undistinguishable from others, making one to think of them as a subset of the whole AMT dataset.

However, the question remains as to whether the above mentioned differences arise as a result of the specific dynamics that generates turbulence (convective rolls) of the CBL in which the Hartman et al. (1997) measurements took place. In any case, it is clear that nominally convective conditions display different behaviours. In fact, even in a well developed CBL a concurrence of shear and buoyancy production mechanisms is actually expected. For instance, the MoninObukhov similarity theory states that for $z \ll|L|$, where $L$ is the Monin-Obukhov length, shear dominates over buoyancy, and it is presumable that data taken at different $z / L$ present a different balance between the two mechanisms. Furthermore, the properties of measurements taken across the CBL depth as a whole could depend on $|L| / z_{i}$, which gives a measure of the fraction of CBL where buoyancy cannot be considered as the only production mechanism.

Going back to data analysis, the two values $\alpha_{0}=2.4$ and $\alpha_{0}=3.3$ in Eq. (3) suggest that the Gaussian case ( $K \rightarrow 3$ as $S \rightarrow 0$ ) may occur for the vertical turbulent velocity, as a transition between the shear and convective production mechanisms (for instance, in the CBL at $z<L_{\mathrm{MO}}$ ).

This observation can be expressed by letting $\alpha_{i}$ be a function of, at least, the Richardson number Ri, thus re-writing Eq. (3) as

$K=\alpha_{0}(\mathrm{Ri})\left(S^{2}+1\right)$

with the constraint $\alpha_{0}(\mathrm{Ri})>1$. It can be argued that $\alpha_{0}$ increases as $z / L_{\mathrm{MO}}$ increases from negative values to zero. No evidence of this can be found in the literature, apart from that provided by Anfossi et al. (1997, their Table 1), which shows an agreement, though very limited (two values only), with this idea.

\section{More on stability effects}

It would be interesting to investigate this issue further if certain details on the measurements were available. In order to overcome the unavailability of such information, we consider a flow over a simple obstacle, and use the analogy between buoyancy and streamline curvature (see, e.g., Bradshaw, 1969; Baskaran et al., 1991) to investigate on the role of stability in determining the character of the $S$ - $K$ relationship.
Table 1. Minimum value of $K$ measured in three different stability conditions.

\begin{tabular}{lll}
\hline$R_{c}<0$ & $R_{c}=0$ & $R_{c}>0$ \\
2.8 & 3.0 & 3.2 \\
\hline
\end{tabular}

In a neutral turbulent flow with streamline curvature, such as a flow over a hill, it is possible to define a curvature Richardson number $\mathrm{Ri}_{\mathrm{C}}$ (Wyngaard, 1967) as

$$
\operatorname{Ri}_{\mathrm{C}}=\frac{\frac{2 U}{R}}{\frac{\partial U}{\partial z}+\frac{U}{R}}
$$

where $U$ is the mean velocity module and $R$ is the streamline curvature radius. Positive $\mathrm{Ri}_{\mathrm{C}}$ corresponds to a dumping term in the turbulent energy budget (e.g., on the hill top) expressed in streamline coordinates, while negative $\mathrm{Ri}_{C}$ represents an unstable contribution, e.g., near the hill base.

An experiment carried out in the Enflo "A" wind tunnel focused attention on third- and fourth-order turbulence statistics. A turbulent boundary layer was generated upstream of a sinusoidal, 2-D hill with aspect ratio $H / L=5$, where $H$ is the obstacle height and $L$ is half the total width. Turbulence was measured with a hot-wire anemometer at 7 different positions streamwise, at 20 vertical levels. Measurements were recorded for times long enough to produce reliable statistics up to the fourth order.

Figure 2 shows data of $S$ and $K$ for three profiles measured far upstream, on the hill top and on the lee side at the hill foot. These regions correspond to $\mathrm{Ri}_{C}=0, \mathrm{Ri}_{\mathrm{C}}>0$ and $\mathrm{Ri}_{\mathrm{C}}<0$, respectively. Apart from the specific structure revealed by the measurements in the unstable case for $S$ in the range 0.2-0.4, the form of the $S$ - $K$ relationship is similar for the three cases and exhibits a minimum value.

We use the minimum as a rough indicator of the different features of the $S$ - $K$ relationship. It seems a reasonable parameter in that it could discriminate data reported by $\mathrm{GH}$ from other data in the AMT dataset.

The minimum measured values of $K$ are reported in Table 1 for three different cases of $\mathrm{Ri}_{C}$ estimated from a similar flow (Maurizi et al., 1997).

Although measured profiles are largely influenced by the specific dynamics of flow over the obstacle and, in particular, by non local equilibrium in the lee side, results are in qualitative agreement with the proposed dependence of expansion coefficients on stability (Eq. 5).

\section{Conclusions}

The $S$ - $K$ relationship is modelled by an expansion in an appropriate space and some parameterisations in literature have 


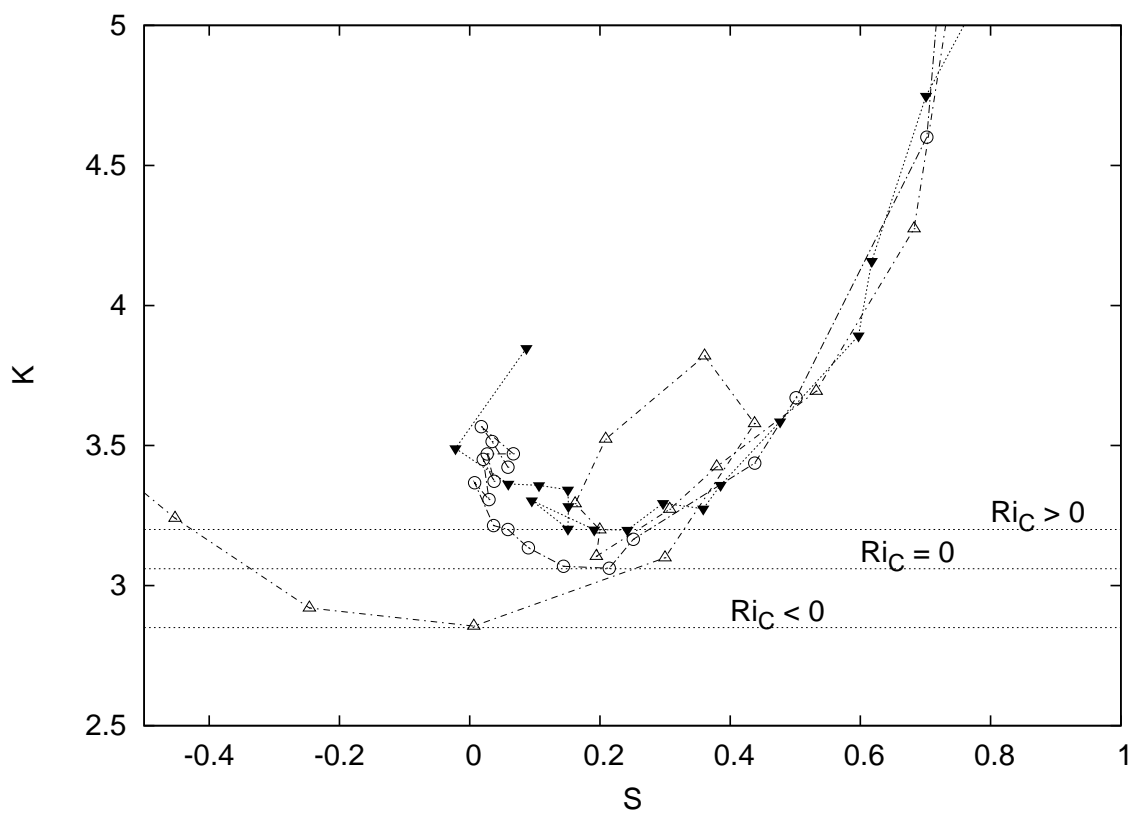

Fig. 2. Data of $S$ and $K$ for vertical velocity measured in the turbulent boundary layer over an obstacle at three different positions $x$ with respect to the obstacle top. Full triangle: $x=0\left(\operatorname{Ri}_{C}>0\right)$, open circle: $x=L\left(\operatorname{Ri}_{C}=0\right)$, open triangle: $x=-\infty\left(\operatorname{Ri}_{C}<0\right)$.

been reduced to this scheme.

In this frame, comparing data for shear- and convectivedominated boundary layers, it is found that model constants should depend on stability. In particular, the Gaussian case can occur in intermediate situations between the purely shear- and purely convective-dominated boundary layer.

Furthermore, an examination of the differences between two datasets for the convective case suggests that that there could be a variety of behaviours for different balances between shear and buoyancy production.

As an example, some data measured in a wind tunnel experiment have shown the possibility that the suggested dependence can be confirmed. It is worth pointing out that this dataset is far from exhaustive in terms of to the problem studied and has been used merely as a indicator.

Further measurements of high-order moments of wind velocity in really different stability conditions are required in order to provide a more quantitative response to the problem.

Acknowledgements. The author would like to thank F. Tampieri for helpful discussions and comments on this work.

Edited by: A. Provenzale

Reviewed by: two referees

\section{References}

Alberghi, S., Maurizi, A., and Tampieri, F.: Relationship between the vertical velocity skewness and kurtosis observed during seabreeze convection, J. Appl. Meteorol., 41, 885-889, 2002.
Anfossi, D., Ferrero, E., Sacchetti, D., and Trini Castelli, S.: Comparison among empirical probability density functions of the vertical velocity in the surface layer based on higher order correlations, Boundary-Layer Meteorol., 82, 193-218, 1997.

Baskaran, V., Smits, A. J., and Joubert, P. N.: A turbulent flow over a curved Hill. Part 2. Effects of streamline pressure gradient, J. Fluid Mech., 232, 377-402, 1991.

Bradshaw, P.: The analogy between streamline curvature and buoyancy in turbulent shear flow, J. Fluid Mech., 36, 179-191, 1969.

Chatwin, P. C. and Sullivan, P.: A simple and unifying physical interpretation of scalar fluctuation measurements from many turbulent shear flows, J. Fluid Mech., 212, 533-556, 1990.

Durst, F., Jovanovic, J., and Kanevce, L.: Probability density distributions in turbulent wall boundary-layer flow, in: Turbulent Shear Flow 5, edited by: Durst, F., Launder, B. E., Lumley, J. L., Schmidt, F. W., and Whitelaw, J. H., Springer, 197-220, 1987.

Gryanik, V. M. and Hartman, J.: A turbulence closure for the convective boundary layer based on a two-scale mass-flux approach, J. Atmos. Sci., 59, 2729-2744, 2002.

Hartman, J., Kottmeier, C., and Raasch, S.: Rolls vortices and planetary boundary-layer development during a cold air outbreak, Boundary-Layer Meteorol., 84, 45-65, 1997.

Kendall, S. M. and Stuart, A.: The Advanced Theory of Statistics, vol. 1, C. Griffin \& Co., London, 4th edn., 1977.

Lenschow, D. H., Mann, J., and Kristensen, L.: How long is long enough when measuring fluxes and other turbulence statistics?, J. Atmos. Oceanic Technol., 11, 661-673, 1994.

Lewis, D. M., Chatwin, P. C., and Mole, N.: Investigation of the collapse of the skewness and kurtosis exhibited in atmospheric dispersion data, Nuovo Cimento C, 20, 385-398, 1997.

Losch, M.: On the validity of the Millionshchikov hypothesis for open-ocean deep convection, Geophys. Res. Lett., 31, L23301, 
doi:10.1029/2004GL021412, 2004.

Maurizi, A. and Lorenzani, S.: On the influence of the Eulerian velocity $p d f$ closure on the eddy diffusion coefficient, BoundaryLayer Meteorol., 95, 427-436, 2000.

Maurizi, A. and Lorenzani, S.: Lagrangian time scales in inhomogeneous non-Gaussian turbulence, Flow, Turbulence and Combustion, 67, 205-216, 2001.

Maurizi, A. and Tampieri, F.: Velocity probability density functions in Lagrangian dispersion models for inhomogeneous turbulence, Atmos. Environ., 33, 281-289, 1999.

Maurizi, A., Trombetti, F., Sabatino, S. D., and Tampieri, F.: Reliability of Third-Order Moments Parameterization for Models of Turbulent Boundary Layer Over Gentle Topography, Nuovo Cimento C, 20C, 273-286, 1997.
Tampieri, F., Maurizi, A., and Alberghi, S.: Lagrangian models of turbulent dispersion in the atmospheric boundary layer, in Ingegneria del vento in Italia 2000. Atti del $6^{\circ}$ convegno nazinale di ingegneria del vento - INVENTO 2000, edited by: Solari, G., Pagnini, L. C., and Piccardo, G., SGEditoriali (Padova), 37-50, 2000.

Wyngaard, J. C.: An experimental investigation of the small-scale structure of turbulence in a curved mixing layer, $\mathrm{PhD}$ thesis, Pennsylvania State University, 1967.

Wyngaard, J. C. and Weil, J. C.: Transport asymmetry in skewed turbulence, Phys. Fluids A, 3, 155-162, 1991. 